2. Breton-Gorius J, Favier R, Guichard J, et al. A new congenital dysmegakaryopoietic thrombocytopenia (Paris-Trousseau) associated with giant platelet alpha-granules and chromosome 11 deletion at 1lq23. Blood 1995; 85: 1805-14.

3. Hart A, Melet F, Grossfeld P, et al. Fli-1 is required for murine vascular and megakaryocytic development and is hemizygously deleted in patients with thrombocytopenia. Immunity 2000; 13: 167-77.

4. Shivdasani RA. Molecular and transcriptional regulation of megakaryocyte differentiation. Stem Cells 2001; 19: 397-407.

5. Spyropoulos DD, Pharr PN, Lavenburg KR, et al. Hemorrhage, impaired hematopoiesis, and lethality in mouse embryos carrying a targeted disruption of the Flil transcription factor. Mol Cell Biol 2000; 20: 5643-52.
6. Bartel FO, Higuchi T, Spyropoulos DD. Mouse models in the study of the Ets family of transcription factors. Oncogene 2000; 19: 6443-54.

7. Raslova H, Komura $\varepsilon$, Le Couédic JP, et al. Fli1 monoallelic expression combined with its hemizygous loss underlies Paris-Trousseau/Jacobsen thrombopenia. J Clin Invest 2004; 114: 77-84.

8. Hanel ML, Wevrick R. The role of genomic imprinting in human developmental disorders: lessons from Prader-Willi syndrome. Clin Genet 2001; 59: 156-64.

9. Schlissel M. Allelic exclusion of immunoglobulin gene rearrangement and expression: why and how? Semin Immunol 2002; 14: 207-12

10. Bix M, Locksley RM. Independent and epigenetic regulation of the interleukin-4 alleles in $\mathrm{CD4}^{+} \mathrm{T}$ cells. Science 1998; 281: 1352-4.
11. Chess A, Simon I, Cedar H, Axel R. Allelic inactivation regulates olfactory receptor gene expression. Cell 1994; 78: 823-34.

12. Penny LA, Dell'Aquila M, Jones MC, et al. Clinical and molecular characterization of patients with distal 1lq deletions. Am J Hum Genet 1995; 56: 676-83.

13. Elowitz MB, Levine AJ, Siggia ED, Swain PS. Stochastic gene expression in a single cell. Science 2002; 297 : 1183-6.

14. Newlands S, Levitt LK, Robinson CS, et al. Transcription occurs in pulses in muscle fibers. Genes Dev 1998; 12: 2748-58.

15. Cook DL, Gerber AN, Tapscott SJ. Modeling stochastic gene expression: implication for haploinsufficiency. Proc Natl Acad Sci USA 1998; 95: 15641-64.

\title{
NOUVELle
}

\section{Pharmacogénomique de l'hormone de croissance: le polymorphisme du récepteur en première ligne}

Pierre Bougnères

> La génétique quantitative fait ses premiers pas chez l'homme. La variabilité individuelle d'un trait humain mesurable (taille, poids, concentration circulante d'un substrat ou d'une hormone...) comporte une part plus ou moins importante de génétique. La variabilité génétique qui différencie les individus repose sur le polymorphisme de leur ADN. Le génome humain comporte environ 14 millions de polymorphismes de «simple nucléotide» (SNP, single nucleotide polymorphism) ainsi que des polymorphismes variés (délétions, microsatellites, minisatellites...). Certains de ces polymorphismes - les uns codants non synonymes, les autres modifiant l'épissage, d'autres encore « régulateurs » - ont des effets fonctionnels. On commence à découvrir le rôle de ces polymorphismes. Certaines variations génomiques jouent un rôle de QTL (quantitative trait locus): celui-ci reste souvent encore une vaste région chromosomique de plusieurs centimorgans [1].

L'action des médicaments offre un vaste champ d'application de la
Service d'Endocrinologie et Inserm U.561, Hôpital SaintVincent-de-Paul, 82, avenue Denfert Rochereau, 75014 Paris, France. pierre.bougneres@ paris5.inserm.fr miques à tester? Pour l'action des médicaments, on peut difficilement recourir à des études de liaison génétique familiales, capables d'identifier des régions (QTL) jusque-là inconnues. En tout cas, c'est impossible pour des «pharmacogénomique » est à la mode [2]. Mais les études dans ce domaine restent rares. Les médicaments ont des effets mesurables. Certains de ces effets reflètent les propriétés thérapeutiques du médicament, d'autres des conséquences indésirables liées à son emploi : par exemple, la croissance en réponse à l'hormone de croissance (GH, growth hormone), et l'insulinorésistance, facteur de diabète, provoquée par la même hormone. Un même variant génomique peut affecter plusieurs traits (pléiotropie). Les effets quantitatifs des variants génomiques peuvent aujourd'hui être évalués in vivo, directement, chez les sujets d'une cohorte de patients traités par le médicament (épidémiogénétique, études d'association). Les effets des variants suspectés induire des différences de réponse individuelle doivent être testés in vitro (génomique fonctionnelle). Comment trouver les variants géno- médicaments très spécifiques, à indications orphelines du type de l'hormone de croissance, qui ne sont administrés qu'à un membre de la famille. Pour rechercher des relations génotype-phénotype, il vaut donc mieux se tourner d'emblée vers des études d'association, directes, entre un variant éventuellement causal et le trait mesurable. Dans le cas qui nous intéresse, le trait quantitatif mesurable est la vitesse de croissance sous traitement par l'hormone de croissance. Quel variant nucléotidique tester? Le premier gène intéressant s'imposait de lui-même. En effet, il ne fallait pas être un grand devin pour imaginer que le récepteur de la $\mathrm{GH}$, porteur d'un polymorphisme fréquent chez les Européens, pouvait moduler les effets physiologiques de la GH. Une étude récente réalisée dans le service d'Endocrinologie de l'hôpital Saint-Vincent-de-Paul (Paris, France) montre que les enfants porteurs 
hétérozygotes ou homozygotes d'une délétion de l'exon 3 du récepteur de la $\mathrm{GH}$ répondent mieux à l'hormone (Figure I) [3]. Ils grandissent plus vite lorsqu'on leur administre de la GH afin de corriger leur petite taille. Les porteurs de

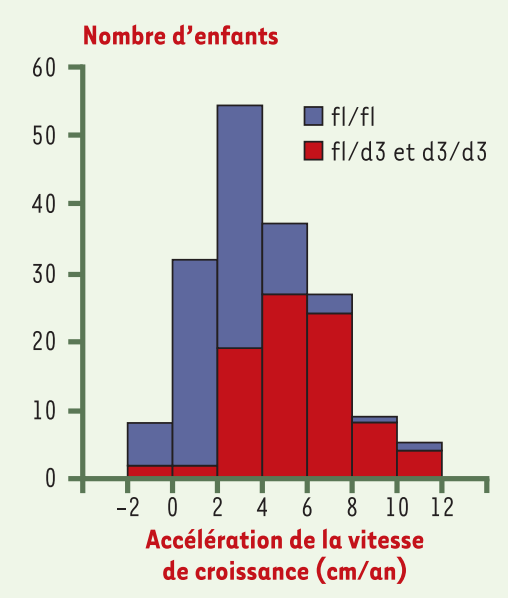

Figure 1. L'accélération de la vitesse de croissance sous $G H$ est dépendante du génotype du récepteur de la $\mathrm{GH}$ ( $\mathrm{d} 3$ : forme délétée). la délétion représentent la moitié de la population européenne: c'est dire que l'effet est important à connaître. II concerne un enfant traité sur deux. L'accélération de la croissance est à peu près le double de celle des homozygotes pour la forme longue du récepteur de la $\mathrm{GH}$, qui composent l'autre moitié de la population. La différence fonctionnelle est nette in vitro, lorsqu'on teste la transduction du signal $\mathrm{GH}$ dans un système de cellules transfectées avec des plasmides porteurs des ADNc des différentes formes du gène GHR. Le gène codant pour le récepteur de la GH est un QTL important pour la croissance humaine. Le polymorphisme impliquant l'exon 3 s'est répandu dans la population européenne, indiquant probablement un avantage sélectif qu'il n'est pas évident d'imaginer [4]. On ne connaît pas précisément sa prévalence en Afrique, continent où il a vu le jour avant la transition hominidés-Homo sapiens, comme le montrent d'élégantes observations du groupe de S. Amselem (Créteil, France). Ce polymorphisme influence fortement la réponse individuelle des patients à l'hormone de croissance. Ces résultats inaugurent d'autres études, dans d'autres domaines thérapeutiques où les réponses sont mesurables et peuvent conduire à l'analyse génétique quantitative. $\diamond$ Pharmacogenomics of the growth hormone: polymorphism of the receptor in the front line

\section{RÉFÉRENCES}

1. Korstanje R, Paigen B. From QTL to gene: the harvest begins. Nat Genet 2002; 31: 235-6.

2. Barton NH, Keightley PD. Understanding quantitative genetic variation. Nat Rev Genet 2002; 3: 11-21

3. Dos Santos C, Essioux L, Teinturier C, et al. A common polymorphism of the growth hormone receptor is associated with increased responsiveness to growth hormone. Nat Genet 2004; 36: $720-4$

4. Pantel J, Machinis K, Sobrier ML, et al, Speciesspecific alternative splice mimicry at the growth hormone receptor locus revealed by the lineage of retroelements during primate evolution. J Biol Chem $2000 ; 275: 18664-9$.

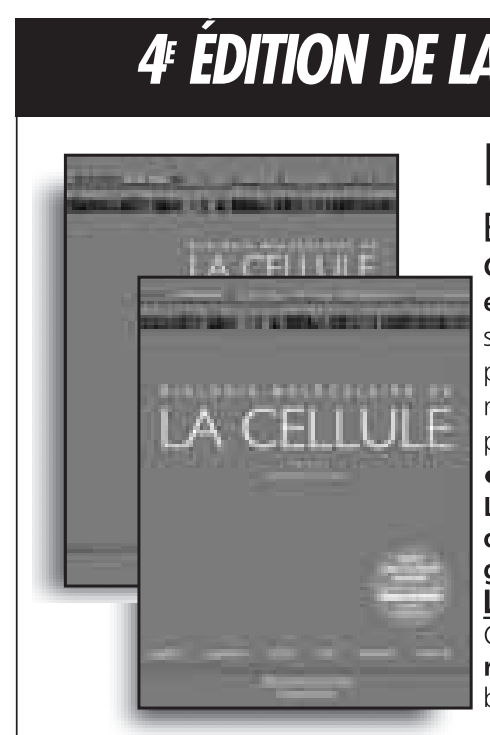

2004. - 4e édition - Collection "Sciences" Broché, 736 pages, 626 illustrations.

\section{BIOLOGIE MOLÉCULAIRE DE LA CELLULE}

Bruce ALBERTS, Alexander JOHNSON, Julian LEWIS, Martin RAF, Keith ROBERTS, Peter WALTER. Cette $4 \mathrm{e}$ édition de l'ouvrage de référence en biologie a été entièrement réécrite, actualisée et augmentée de 6 chapitres de génétique moléculaire totalement inédits et d'un nouveau chapitre sur les germes pathogènes, l'infection et l'immunité. Complet, moderne, il est divisé en 5 grandes parties - les principes élémentaires de biologie cellulaire et la biochimie fondamentale $\bullet$ les mécanismes génétiques de base, l'expression et la transmission des informations génétiques $\bullet$ les principales méthodes expérimentales d'étude des cellules • l'organisation interne des cellules - le comportement des cellules dans les organismes multicellulaires.

Le CD-Rom offert dans l'ouvrage, la richesse des 2100 illustrations, les résumés en fin de chapitre, la rédaction très didactique, la mise en valeur des termes à retenir et l'important glossaire ajoutent à l'extraordinaire accessibilité du livre.

LIVRE D'EXERCICES - John WILSON ef Tim HUNT

Complémentaire du livre de cours, un outil indispensable à l'étudiant pour préparer, réviser, et réussir l'épreuve de biologie cellulaire. : 1388 problèmes, leurs corrigés, 12 pages de références bibliographiques utiles et un index de 6000 entrées. 2004 - Broché, 480 pages et 450 illustrations

Ces ouvrages sont en vente séparément ou en " pack spécial-étudiant " - Livre de cours + Livre d'exercices - au prix exceptionnel de $150 €$ au lieu de $180 €$.

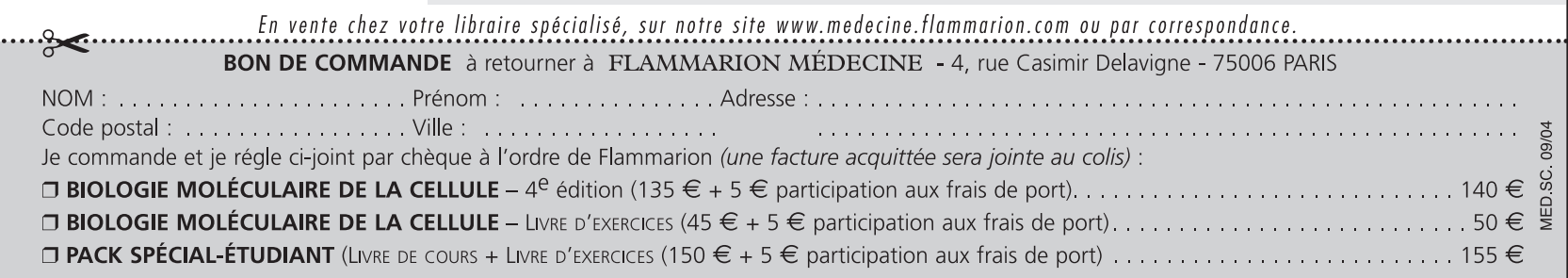

